\title{
Microstrip Patch Antenna Miniaturization Using Magneto-Dielectric Substrates for Electromagnetic Energy Harvesting
}

\author{
Taher AlSharabati
}

\begin{abstract}
A mathematical modelling, and a derivation of the main parameters of the Magneto - Dielectric materials (substrate) and their effect on microstrip patch antenna design is shown. The Magneto - Dielectric materials (substrate) is shown to miniaturize the antenna size and enhance the bandwidth when used in the design of the microstrip patch antenna. The progression of the foundational modelling starts with laying out the concepts of the ferrimagnetic materials in terms of their permeability and permittivity, the components of antenna miniaturization. First, a ground free elliptical microstrip patch antenna (GFDSEPA) is simulated for miniaturization purposes at the $900 \mathrm{MHz}$ cellular band. A size reduction of almost $50 \%$ as well as bandwidth enhancement $(100 \%)$ is achieved by utilizing the GFDSEPA. More size reduction is achieved by employing the magneto - dielectric structure; in this case the commercially available Rogers MAGTREX 555 substrate is used. Other performance parameters show comparable results between the antenna simulated based on dielectric only substrate and the one based on magneto-dielectric substrate. A comparison of the main parameters between the results of this work and other results in the literature is shown. The application of the microstrip patch antenna design in energy harvesting, by using a rectifier circuit, is shown. Layout scenarios of the energy harvester are proposed. The proposed layout of the energy harvester ensures practicality of the proposed design and assures correlation between simulation results and experimental results.
\end{abstract}

Index Terms-Electromagnetic energy harvesting, microstrip patch antenna, antenna return loss, plane radiation, antenna gain, antenna impedance.

\section{INTRODUCTION}

Some research into energy harvesting has gained great momentum in recent years due to the need for alternative and available energy sources. For example, electric energy is the most used type of energy. Electromagnetic energy harvesting is an alternative source of DC power needed to supply low voltage devices.

Microstrip antennas have found many applications in the wireless products, ranging from GPS to sensors, RFID to WiFi and many applications. Their versatility, reconfigurability and

Manuscript received November 26, 2020; revised February 18, 2021. Date of publication May 19, 2021. Date of current version May 19, 2021.

Author is with the United States Patent and Trademark Office (USPTO) (email: talsharabati@uspto.gov).

Digital Object Identifier (DOI): 10.24138/jcomss-2020-0005 ease of fabrication made them very attractive from a product point of view. They are made of metal conductor sheets (often copper) with many different shapes built on a dielectric or composite magnetic and dielectric (called magneto-dielectric) substrates.

Challenges that face the design of microstrip patch antennas include, but not limited to, efficiency: as efficient as possible, and size; as small as possible: miniaturization. For a normal dielectric material substrate, these might be conflicting goals.

While for a magneto-dialectic (MD) substrates/material, this is deemed possible.

For example, in [1], barium hexaferrite (BaFe12O19) magneto - dielectric material was used as a substrate to miniaturize the microstrip patch antenna for radio frequency sensor applications. The amount of reduction in size reached up $42.5 \%$ as opposed to the dielectric only substrate, FR4. Their electromagnetic characterization achieved a complex permittivity of $(\varepsilon=6.2-\mathrm{j} 0.04)$ and complex permeability $(\mu=1.9-\mathrm{j} 0.18)$.

In [2], the magneto - dielectric material, Nickel Zinc $\mathrm{Ni}_{0.5} \mathrm{Zn}_{0.5} \mathrm{Fe}_{2} \mathrm{O}_{4}$, was used for body worn sensor applications on a flexible material. The magneto - dielectric composite achieved miniaturization for the rectangular shaped antenna and enhanced its bandwidth. The Nickel Zinc as a substrate reduced the size of the antenna and enchanted the bandwidth by $49.62 \%$. The magneto-dielectric substrate performed better than the dielectric only substrate. As an added benefit the magnetodielectric material reduced the amount of coupling between the body and the antenna system.

In [3], an asymmetric star shaped approach was used to design a slotted microstrip patch antenna to achieve both size reduction and cross polarization for a UHF-RFID based sensor antenna application. The size reduction achieved is roughly $20 \%$ over conventional microstrip antennas. Resonance is achieved by changing the size of the four stars: S1, S2, S3 and $\mathrm{S} 4$, positioned in the middle of the quadrants of the patch, and therefore changing the current distribution of the patch.

In [4], the idea of a cuboid shape ridge in size reduction was used. A semicircle arc was projected and used to miniaturize a circular microstrip patch antenna.

In [5] more size reduction was achieved. The magnetodielectric material was used as a substrate for a UHF-RFID based sensor antenna. The use of the Magneto-Dielectric 
substrate reduced the size of the proposed sensor by about $75 \%$ as compared to conventional (dielectric only) substrates. In [19], a $70 \%$ size reduction was achieved, at $2.4 \mathrm{GHz}$, in designing a rectangular microstrip patch antenna (to obtain circular polarization) by using a two-dimensional magneto-dielectric substrate over using conventional substrate (dielectric only) microstrip patch antenna. No bandwidth enhancement was achieved due to increased magnetic permeability. The authors achieved a $75 \%$ in size reduction by using an artificially twodimensional metamaterial (ATDM) substrate (based on Rogers RO4003 substrate as the host substrate) in [20]; not a magnetodielectric substrate. However, the ATDM in [20] seems very complex and the extra 5\% size reduction does not justify the complexity. In [21], the host substrate was magneto-dielectric one for the ATDM material. They were able to achieve $74.83 \%$ (at $2.35 \mathrm{GHz}$ ) size reduction as compared with the conventional antenna on host dielectric It should be mentioned that both [20] and [21] use the split-ring resonator technique to achieve effective permeability and permittivity greater than that of the host dielectric material to achieve the size reduction. In [22], another artificial magneto-dielectric resonator antenna (AMDRA) based on split ring resonator (SRR) was used to achieve both low loss and miniaturization at $3.857 \mathrm{GHz}$. The low loss was achieved by using metallic inclusions. In [23], The magneto-dielectric substrate was used to achieve miniaturization of $77 \%$, for the microstrip patch antenna, over a microstrip patch antenna designed over a dielectric substrate only. The target application was RFID in the UHF band.

Magneto-dielectric materials found applications in flexible wearable devices due to the size constraint those devices demand. In [6], a flexible magnetodielectric polymer-based nanocomposite layer covering a Kapton (tape) substrate was used to design a flexible antenna. The design achieved multi band operation and covered two frequency ranges: [1.8-2.45] $\mathrm{GHz}$ and [5.15-5.825] GHz.

The paper is organized as follows: section II goes over the background and theory of Magneto-dielectric materials. In addition, it goes over theoretical modelling and the background affecting the different parameters of the antenna design such as radiation conductance, bandwidth, and radiation efficiency. Section III goes over the design of the ground free elliptical microstrip patch antenna, matching, rectifier circuit and simulation (results) of all antenna structures including the one (proposed) of the Magneto-dielectric materials along with a comparison between the results of this paper and [14]. Section IV has the conclusion and recommendations.

\section{MiniATURIZATION AND BANDWIDTH ENHANCEMENT USING MAGNETO-DiELECTRIC MATERIALS}

Magneto-dielectric materials are composites of ceramic, ferrimagnetic materials or ferrimagnetic compounds (also known as ferrites [8]) and dielectric materials. These Magnetodielectric materials offer advantages, when used as substrates for patch antennas, in terms of size (miniaturization), increased bandwidth and higher antenna efficiency [9].

Ferrimagnetic compounds are characterized by their permeability tensor, $\mu$. Table I shows the symbols used with their descriptions. The general tensor characterization for any anisotropic material is:

$$
[\mu]=\left[\begin{array}{lll}
\mu_{x x} & \mu_{x y} & \mu_{x z} \\
\mu_{y x} & \mu_{y y} & \mu_{y z} \\
\mu_{z x} & \mu_{z y} & \mu_{z z}
\end{array}\right]
$$

for the $z$-bias condition, the above equation reduces to:

$$
[\mu]=\left[\begin{array}{ccc}
\mu_{x x} & j \kappa & 0 \\
-j \kappa & \mu_{y y} & 0 \\
0 & 0 & \mu_{o}
\end{array}\right]
$$

where $\mu_{o}$ is the permeability of free space, and

$$
\kappa=\mu_{o} \frac{\omega \omega_{m}}{\omega_{o}^{2}-\omega^{2}}
$$

$\omega_{o}$ is the precession frequency [1];

$$
\begin{aligned}
& \omega_{o}=\mu_{o} \gamma H_{o} \\
& \omega_{m}=\mu_{o} \gamma \mathcal{M}_{s}
\end{aligned}
$$

$\gamma$ is the gyromagnetic ratio $\left(1.759 \times 10^{11} \mathrm{C} / \mathrm{kg}\right), H_{o}$ is the applied bias field and $\mathcal{M}_{s}$ is the saturation magnetization for the magnetic moment, $\mathcal{M}$, and is a constant for each ferrite material. The applied bias field, $H_{o}$, is related to $E_{o}$ by [10],

$$
H_{o}=j \sqrt{\frac{\varepsilon_{r} \varepsilon_{o}}{\mu_{o} \mu_{r}}} E_{o}
$$

\begin{tabular}{|c|c|c|}
\hline & Description & Unit of measure \\
\hline$\varepsilon$ & complex permittivity & \\
\hline$\mu$ & complex permittivity & \\
\hline$\omega_{o}$ & precession frequency & \\
\hline$\gamma$ & gyromagnetic ratio & $1.759 \times 10^{11} \mathrm{C} / \mathrm{kg}$ \\
\hline $\mathcal{M}_{s}$ & saturation magnetization & \\
\hline $\mathrm{G}$ & Gain of Antenna & $\mathrm{dB}$ \\
\hline $\mathrm{D}$ & Directivity of Antenna & $\mathrm{dB}$ \\
\hline$d$ & height of the substrate & $\mathrm{m}$ \\
\hline$W$ & width of the patch & $\mathrm{m}$ \\
\hline$\kappa$ & gyro tropic material & \\
\hline $\mathcal{M}$ & magnetic moment & \\
\hline$H_{o}$ & bias field & $\mathrm{A} / \mathrm{m}$ \\
\hline$F_{o}$ & resonant frequency & $\mathrm{Hz}$ \\
\hline$G_{r}^{\text {patch }}$ & radiation conductance & \\
\hline$B W$ & bandwidth & $\mathrm{Hz}$ \\
\hline$\eta_{\text {rad }}$ & radiation efficiency & \\
\hline $\tan \delta$ & loss tangent & \\
\hline $\tan \delta_{\varepsilon}$ & dielectric loss tangent & \\
\hline $\tan \delta_{\mu}$ & magnetic loss tangent & \\
\hline$\eta_{\text {Ant }}$ & antenna efficiency & \\
\hline
\end{tabular}

TABLE I

LIST OF SYMBOLS USED WITH THEIR DESCRIPTION AND UNITS (WHEN APPLICABLE)

Just as ferrimagnetic materials have permeability tensors, dielectric materials have permittivity tensors, $[\mathcal{E}]$, where 


$$
[\varepsilon]=\left[\begin{array}{lll}
\varepsilon_{x x} & \varepsilon_{x y} & \varepsilon_{x z} \\
\varepsilon_{y x} & \varepsilon_{y y} & \varepsilon_{y z} \\
\varepsilon_{z x} & \varepsilon_{z y} & \varepsilon_{z z}
\end{array}\right]
$$

So, a magneto-dielectric material has both a permeability tensor and a permittivity tensor.

The complex permittivity is

$$
\varepsilon=\varepsilon^{\prime}-j \varepsilon^{\prime \prime}
$$

of the dielectric medium, which contains complex electric susceptibility, accounts for the dielectric material loss in the magneto-dielectric substrate.

Similarly, the complex permeability is

$$
\mu=\mu^{\prime}-j \mu^{\prime \prime}
$$

contains the complex magnetic susceptibility and accounts for the magnetic loss of the magneto-dielectric substrate.

In equation (8) above, the real part is

$$
\varepsilon^{\prime}=\varepsilon_{r} \varepsilon_{o}
$$

and in equation (9) above, the real part is

$$
\mu^{\prime}=\mu_{r} \mu_{o}
$$

Similarly, for the imaginary part

$$
\varepsilon^{\prime \prime}=\frac{\omega \varepsilon^{\prime} \tan \delta_{\varepsilon}-\sigma}{\omega} .
$$

Similarly

$$
\mu^{\prime \prime}=\frac{\omega \mu^{\prime} \tan \delta_{\mu}-\sigma}{\omega}
$$

where, $\tan \delta_{\varepsilon}$ is the dielectric loss tangent and, $\tan \delta_{\mu}$, is the magnetic loss tangent.

The frequency dependence of $\mu$ ” on frequency comes from the relation [9]

$$
\int_{0}^{\infty} f \mu^{\prime \prime}(f) d f=k_{a} \frac{\pi}{2}\left(2 \gamma \mathcal{M}_{s}\right)^{2}
$$

which is a generalization of Snoek's law

$$
\left(\mu_{o}^{\prime}-1\right) F_{o}=\frac{4}{3} \gamma \mathcal{M}_{s}
$$

where $F_{o}$ is the resonant frequency.

\section{A. Radiation Conductance}

In terms of radiation conductance, [10] treated it in both the elevation angle and the azimuth angle directions, while [11] treated it only in the elevation angle direction. In [10], the radiation conductance is

$$
\begin{gathered}
G_{r}^{\text {patch }}=\frac{4}{377 \pi^{2}} \iint_{0}^{\pi}\left[\frac{\sin \left(\frac{k_{o} W \cos \theta}{2}\right)}{\cos \theta} \cos \left(\frac{k_{o} \sin \theta \sin \varphi}{2 k_{g}}\right)\right]^{2} \\
\sin ^{3}(\theta) d \theta d \phi
\end{gathered}
$$

while in [11],

$$
G_{r}^{p a t c h}=\frac{\left[\frac{\pi W}{\lambda}\right]^{2}}{377 \pi} \int_{0}^{\pi} \frac{\sin ^{3}(\theta)}{\cos ^{2} \theta} d \theta
$$

It must be mentioned that [11] followed the simplistic approach of zero order theory in their treatment of the radiation conductance. The radiation conductance is used to calculate the radiation quality factor [13] and its contribution to the stored energy in the patch antenna.

\section{B. Bandwidth and Radiation Efficiency}

Expanding the scope of analysis above leads to expressions for bandwidth and radiation efficiency; $\eta_{\text {rad }}[6]$ :

$B W=\frac{1}{\sqrt{2}}\left(240 \frac{d}{W} G_{r}^{p a t c h} \sqrt{\frac{\mu_{r}}{\varepsilon_{r}}}+\tan \delta_{\varepsilon}+\tan \delta_{\mu}\right)$

and,

$$
\eta_{\text {rad }}=\frac{1}{1+\frac{\tan \delta_{\varepsilon}+\tan \delta_{\mu}}{240 G_{r}^{\text {patch }}}\left(\frac{W}{d}\right) \sqrt{\frac{\mu_{r}}{\varepsilon_{r}}}}
$$

where $d$ is the height of the substrate, $W$ is the width of the patch. The antenna efficiency; $\eta_{A n t}$ is related to the gain $G$ and directivity, $D$ :

$$
\eta_{A n t}=\frac{G}{D}
$$

\section{ANTENNA AND GFDSEPA}

Designing a patch antenna at the $900 \mathrm{MHz}$ cellular band is not common due to the large size the antenna occupies as opposed to the size of the antennas for higher bands. The $900 \mathrm{MHz}$ band antenna is the largest out of most wireless bands in terms of size even with the best miniaturization techniques developed recently. However, the $900 \mathrm{MHz}$ cellular band has the largest potential ambient power. The higher the frequency is, the lower the ambient power. This is due to the power constraints the standards bodies stipulate on the transmitted power of each band and each service. Based on this, one would guess that the energy harvested from the cellular $900 \mathrm{MHz}$ band would be, potentially, the largest compared to other bands.

Circular polarization is achieved when the electric field or magnetic field vectors are composed of two, equal in magnitude but orthogonal linear components [7]. The orthogonality is in multiples of 90 degrees. To achieve cross polarization, the slightly elliptical and tilted antenna configuration was used as shown in Fig. 1. Total dimensions are $132.5 \times 162.7 \mathrm{~mm}$ on a Roger's Duroid 5870 substrate $\left(\varepsilon_{r}=2.33\right.$, $\tan \delta=0.0005$, height, $h=3.175 \mathrm{~mm}$, and conductor thickness, $t=35 \mu \mathrm{m})$. An open stub of size $40 \times 3 \mathrm{~mm}$ was employed for matching purposes. 


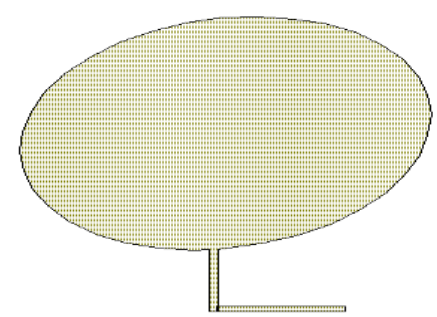

Fig. 1. Geometry of simulated antenna.

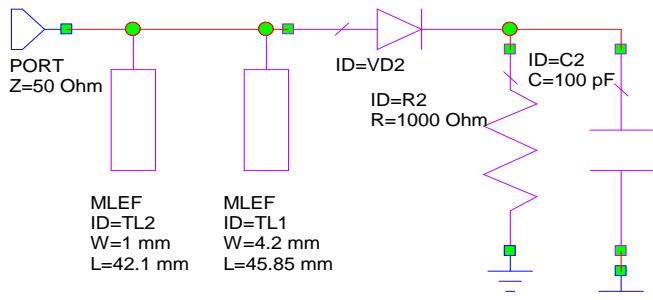

Fig. 2. Simulated matching and simple diode rectifier circuit.

\section{A. Design of Matching Circuit and Rectifier}

Fig. 2 shows the matching circuit with the rectifier used. The rectifier circuit is a single detector zero bias Schottky diode. The two open stubs are used to bring the input impedance of the rectifier to 50ohms for matching purposes.

\section{B. Simulation Results}

A gain of $4.73 \mathrm{~dB}$ was obtained at mid band as shown in Fig. 3. A return loss $(\mathrm{RL})$ of $-43 \mathrm{~dB}$ and $-30.29 \mathrm{~dB}$ was obtained for the antenna and matching circuit respectively as shown in Fig. 4. Fig. 5 shows the simulated gain in linear polarization as well as the right hand and left-hand circular. Fig. 6 shows the 3D gain radiation pattern while Fig. 7 shows one of the possible ways the matching circuit and the rectifier circuit could be laid out on a printed circuit board. The two open stub matching circuit adds to the size of the whole energy harvesting system.

\section{C.Ground Free Double Stub Elliptical Patch Antenna (GFDSEPA)}

Removing the ground plane leads to reduced antenna size. Fig. 8 shows the reduced antenna size when the ground plane is removed.

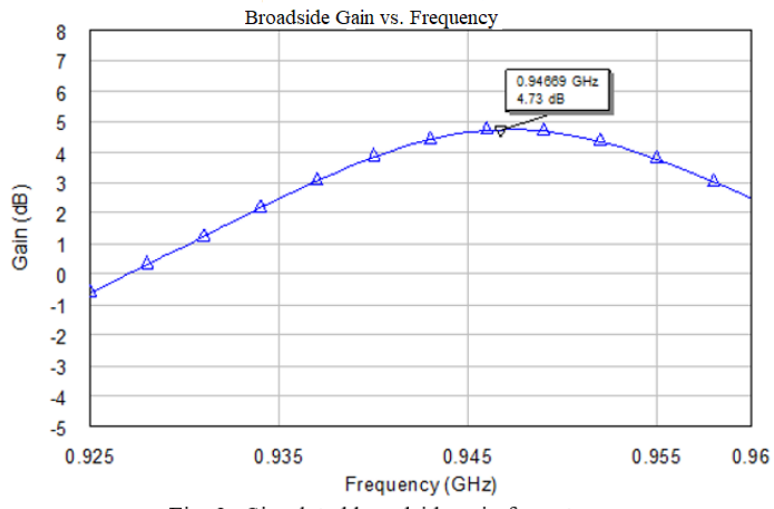

Fig. 3. Simulated broadside gain for antenna.

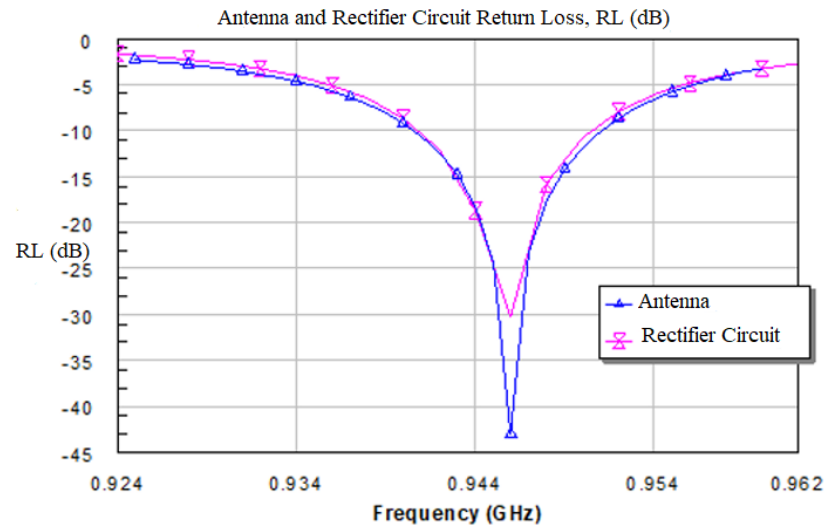

Fig. 4. Simulated return loss for antenna, matching circuit, and rectifier.

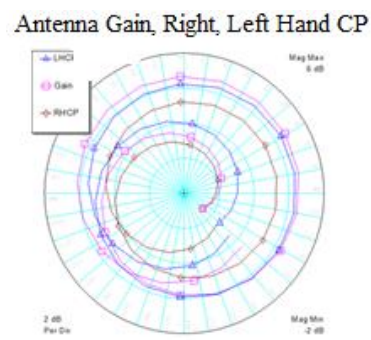

Fig. 5. Simulated gain, right hand, and left-hand circular polarization.

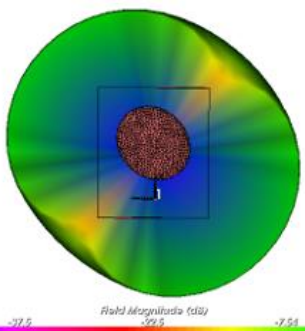

Fig. 6. Simulated radiation 3D pattern.

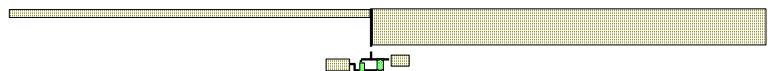

Fig. 7. Possible layout scenario of matching and rectifier circuits in Fig. 2.

Total dimensions are $7.83 \times 7.6 \mathrm{~cm}$ for the same substrate; a size reduction of almost $50 \%$. However, the gain and circular polarization suffered as Figs. 9 and 11 show. Fig. 10 shows the simulated return loss for the antenna and the combination of the matching and rectifier circuits shown in Fig. 15. Fig. 10 indicates the improvement in return loss and bandwidth. Compared to Fig. 4. The RL improved by about $2 \mathrm{~dB}$ while the bandwidth increased by $100 \%$. Figs. 12 \& 13 show the 2 -D Efield and $\mathrm{H}$-field patterns. They show co-polarization and cross polarization patterns of the antenna radiation and gain, reflecting the $3 \mathrm{D}$ radiation patterns shown in Fig. 14 and asserting the omni-directional operation of the antenna. Fig. 16 shows a possible layout scenario for the matching and rectifier circuits considering (as much as possible) component orientation for reduced coupling effects. Fig. 14 shows the 3D radiation pattern. Since the ground plane is removed and the bottom boundary (just like the top boundary) of the antenna 
became virtually infinite open, the radiation pattern became of that (almost) omni-directional. Except for a hollow slit in the middle, relative to the thickness of the substrate, the $360^{\circ}$ omnidirectional pattern is evident. Due to the omni-directional pattern, the directivity is reduced and therefore the gain is lowered to slightly over $2 \mathrm{~dB}$ and the $\mathrm{CP}$ is lowered to almost $0.4 \mathrm{~dB}$. Reducing the antenna size makes it possible to use a better rectifier circuit for better conversion efficiency. The room saved from reducing the antenna size could be used to employ more complex and more efficient rectifier circuit such as the one used in [25] and is shown in Fig. 15.

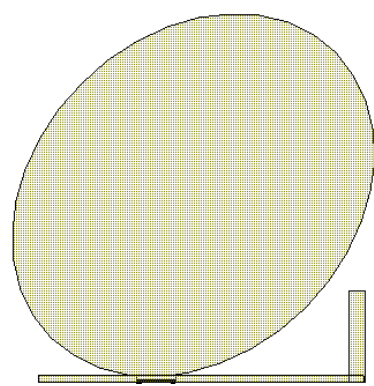

Fig. 8. Optimized geometry of simulated GFDSEPA on a Roger's Duroid 5870 Substrate.

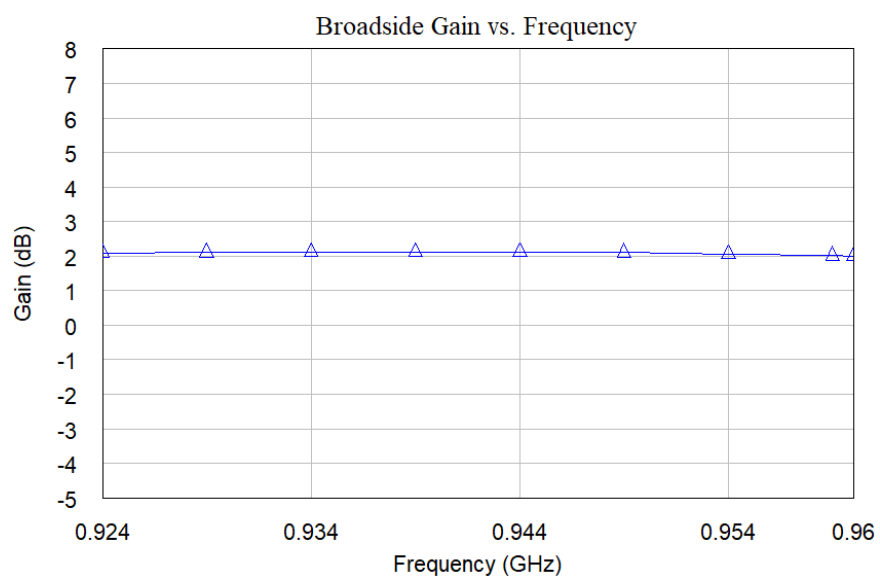

Fig. 9. Simulated broadside gain of the GFDSEPA.

\section{Rogers MAGTREX 555 Laminate.}

The Rogers' MAGTREX 555 [10] is an example of a magneto dielectric substrate available for commercial use. Its data sheet is available on the public domain. Based on the datasheet,

$$
[\mu]=\left[\begin{array}{ccc}
6.0 & j \kappa & 0 \\
-j \kappa & 6.0 & 0 \\
0 & 0 & \mu_{o}
\end{array}\right]
$$

and,

$$
[\varepsilon]=\left[\begin{array}{lll}
6.5 & \varepsilon_{x y} & \varepsilon_{x z} \\
\varepsilon_{y x} & 6.5 & \varepsilon_{y z} \\
\varepsilon_{z x} & \varepsilon_{z y} & 5.3
\end{array}\right]
$$

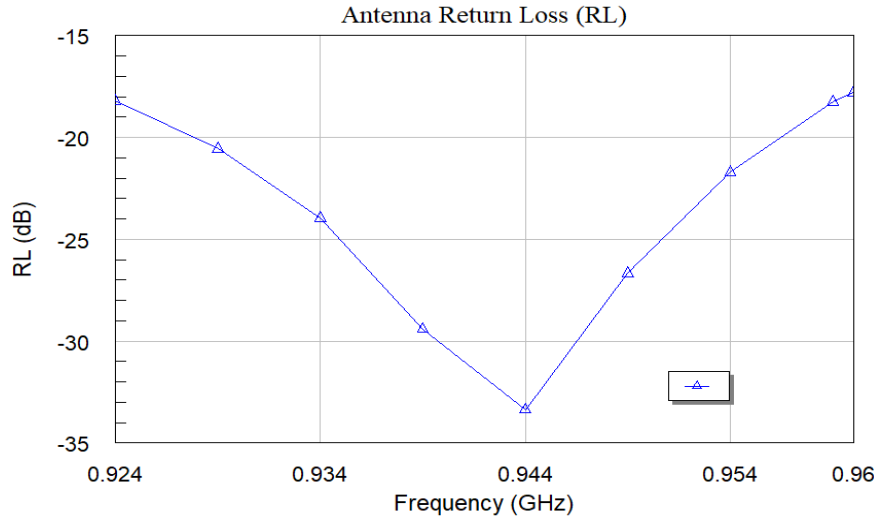

Fig. 10. Simulated return loss of the GFDSEPA, matching circuit and rectifier used.

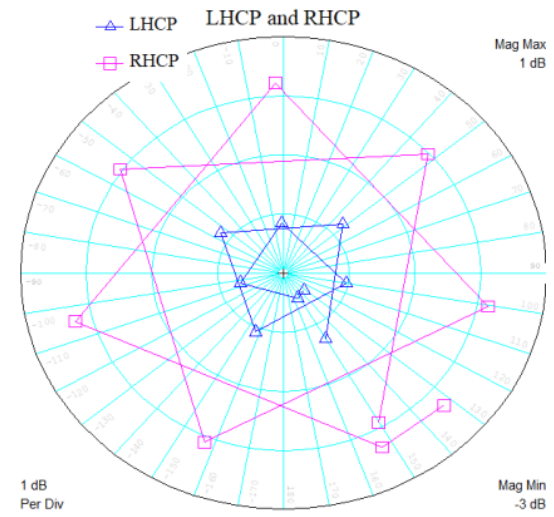

Fig. 11. Simulated gain, right hand, and left-hand circular polarization of GFDSEPA.

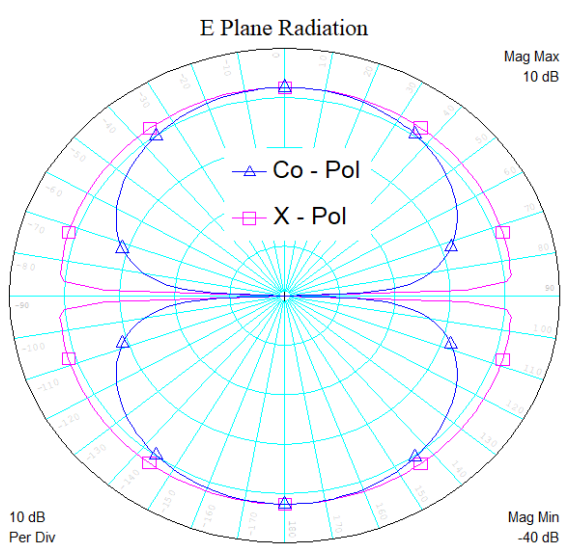

Fig. 12. Simulated 2D E-field pattern of the GFDSEPA.

Fig. 17 shows the simulated antenna on MD substrate. The same design approach used in Fig. 8 is used here i.e. the same 45 degree tilted elliptical structure with two open stubs used for 50 -ohm matching purposes. The resulting return loss is shown in Fig. 19 while the 3D radiation field intensity is shown in Fig. 20. Fig. 21 displays the results of circular polarization while Fig. 22 displays the 2-D elevation radiation. Table II shows a comparison made between the results of this work and the results of the work done in [14]. 


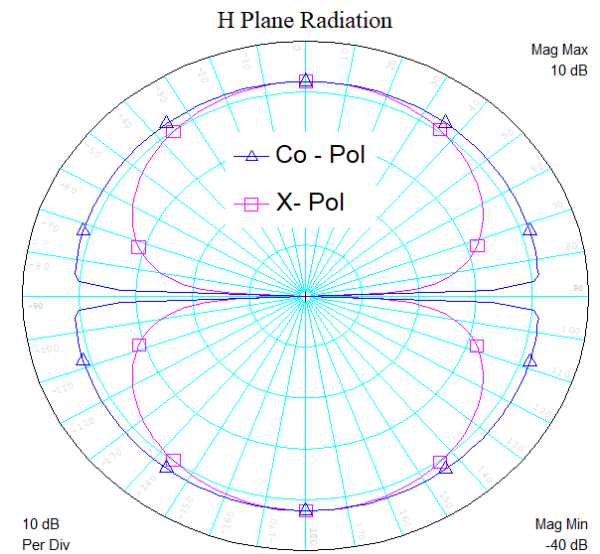

Fig. 13. Simulated 2D H-field pattern of the GFDSEPA

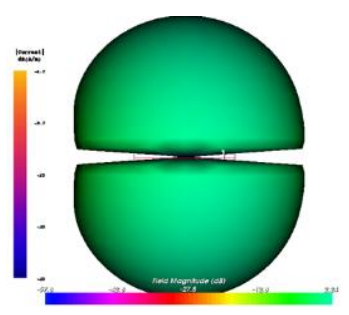

Fig. 14. Simulated 3D radiation pattern of the GFDSEPA.

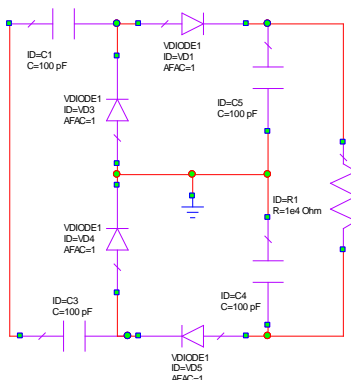

Fig. 15. Simulated modified full wave Greinacher rectifier used in [25].

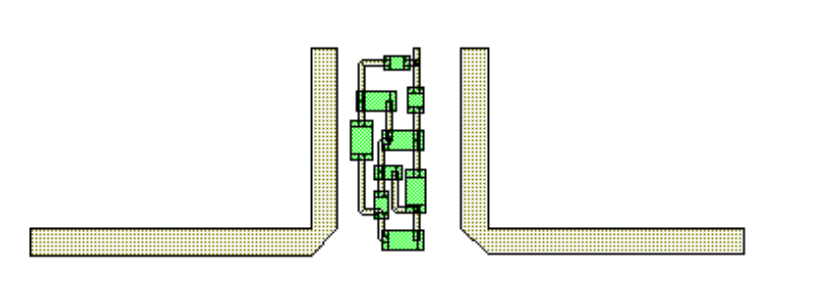

Fig. 16. A possible layout scenario for the matching circuit and the rectifier circuit (shown in Fig. 15) of GFDSEPA.

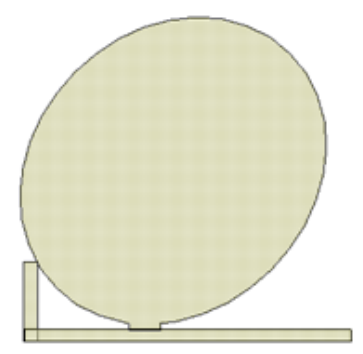

Fig. 17. Patch Antenna Simulated on a Rogers' MAGTREX 555 Magneto Dielectric Substrate.

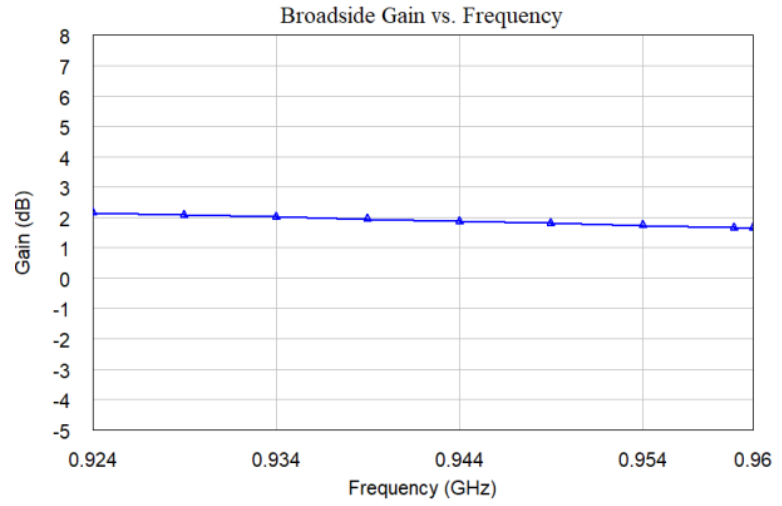

Fig. 18. Gain of Patch Antenna in Fig. 17 with antenna efficiency of $80 \%$ and radiation efficiency of $99.97 \%$.

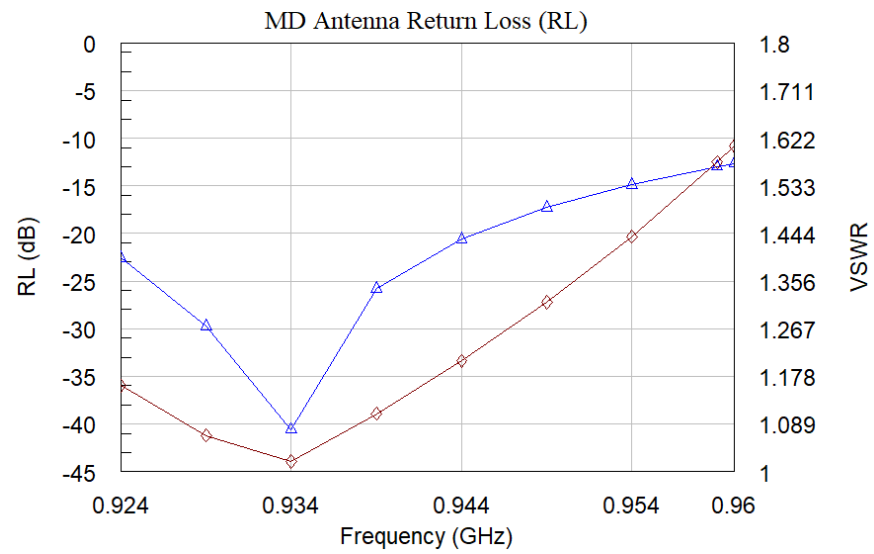

Fig. 19. Return Loss (RL); Left, and VSWR; Right, of Patch Antenna in Fig. 17.

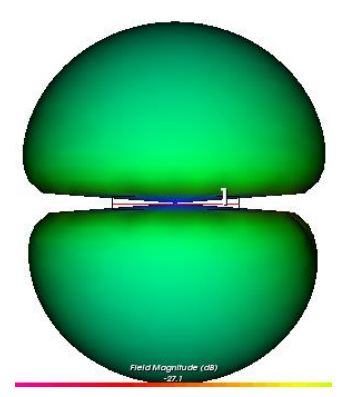

Fig. 20. 3D Radiation Pattern of Patch Antenna in Fig. 17.

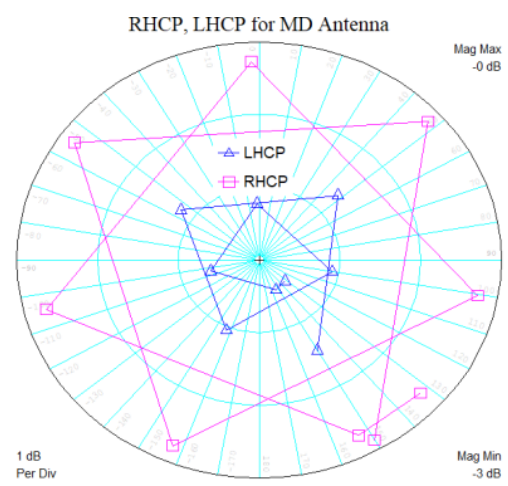

Fig. 21. Circular Polarization of the MD Patch Antenna 


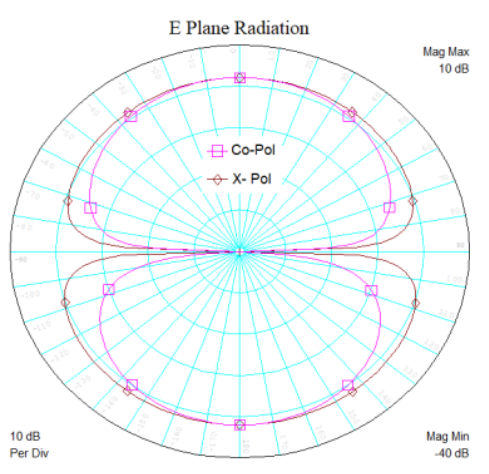

Fig. 22. 2-D Elevation ( $\boldsymbol{\theta})$ Radiation of the MD Patch Antenna.

TABLE II

COMPARISON BETWEEN THIS WORK AND [14].

\begin{tabular}{|c|c|c|c|c|c|c|c|}
\hline Ref. & $\tan \delta$ & $\varepsilon_{r}$ & $\begin{array}{c}h \\
(\mathrm{~mm})\end{array}$ & $\begin{array}{c}\text { Gain } \\
(\mathrm{dB})\end{array}$ & $\begin{array}{c}\text { Size } \\
(\mathrm{cm})\end{array}$ & $\mathrm{RL}_{\min }(\mathrm{dB})$ & $\begin{array}{c}\mathrm{BW} \\
(\mathrm{MHz})\end{array}$ \\
\hline$[14]$ & 0.0027 & 3.55 & 0.8 & 1.46 & $4.6 \times 3.0$ & -21 & 12 \\
\hline $\begin{array}{c}\text { Fig. } \\
8\end{array}$ & 0.0005 & 2.33 & 3.175 & 2.1 & $8.14 \times 8.0$ & -33.37 & $\begin{array}{c}36 \\
(100 \%)\end{array}$ \\
\hline $\begin{array}{c}\text { Fig. } \\
17\end{array}$ & $(1)$ & $(1)$ & 3.56 & 2.2 & $6.16 \times 6.2$ & -40.61 & $\begin{array}{c}36 \\
(100 \%)\end{array}$ \\
\hline
\end{tabular}

${ }^{1)}$ Anisotropic material

\section{CONCLUSION AND DISCUSSION}

In this paper, a size-reduction antenna configuration is simulated for the $900 \mathrm{MHz}$ cellular band. The tilted elliptical shape antenna is simulated on a Roger's Duroid 5870 substrate. The size reduction was achieved by removing the ground plane beneath the antenna. The results obtained are acceptable given the expected higher power levels for the $900 \mathrm{MHz}$ band available for energy harvesting. The reduction in size obtained is almost 50\%. Proposed layout scenarios for both cases; with ground plane and ground plane free antenna with matching circuit configurations are also shown. More size reduction was achieved by using the commercially available magneto dielectric substrate. The ground free magneto - dielectric antenna not only presented bandwidth and efficiency enhancement, like the dielectric only version, it also achieved miniaturization by reducing the size more than $50 \%$. Table II shows a comparison between the performance of the two antennas and the results in [14]. The comparison shows tradeoffs in performance between the three designs. Depending on the performance criteria, these designs have advantages and disadvantages. Fig. 16 can be used along with Fig. 17 to complete the layout of the electromagnetic energy harvesting process to produce a DC voltage that can be boosted/amplified by using a DC/DC boost convertor to drive low battery, low voltage devices.

Different miniaturization and bandwidth enhancement techniques could be researched to see how the combination of these techniques with the MD materials could lead to further reducing the size of the patch antenna and maintaining maximum bandwidth efficiency. In addition, gain and efficiency are not compromised. For example, the miniaturization technique used in [17], which is based on fractal geometries, could be implemented on the MD substrate to achieve further miniaturization, more than $37.25 \%$. The combination of fractal geometries and MD substrate could also lead to higher degrees of freedom in resonant frequency rather than restricting it to the lower frequency side [17]. Also, the Cshaped microstrip patch antenna with partial ground plane in [18] could be investigated to be used on the MD substrate to further enhance the bandwidth while keeping the good results for the return loss and VSWR.

Previous work, [24], showed strong correlation between the simulation results using the $3 \mathrm{D}$ simulation tool along with its layout engine and the measured results of the manufactured/fabricated printed circuit board. The results of the manufactured/fabricated printed circuit board are in terms of gain and return loss measurements. The strong correlation /conclusion makes the simulation results in this paper very credible if they were to be matched with the experimental results. It should be mentioned the antenna designed in this work could be used in other applications other than the electromagnetic energy harvesting. For example, it could be used in RFID tags and reader/scanner applications where the size of the antenna could be further reduced especially when extending the operating frequency in the $[5-8] \mathrm{GHz}$ range.

\section{REFERENCES}

[1] Saini, Ashish, et al. "Miniaturization and Bandwidth Enhancement of a Microstrip Patch Antenna Using Magneto-Dielectric Materials for Proximity Fuse Application.” Journal of Electronic Materials, vol. 46, no. 3, 2017, pp. 1902-1907., doi:10.1007/s11664-016-5256-0.

[2] Gogoi, Pragyan Jyoti, et al. "CPW-Fed Body Worn Monopole Antenna on Magneto-Dielectric Substrate In C-Band.” Progress in Electromagnetics Research C, vol. 84, 2018, pp. 201-213., doi:10.2528/pierc18040604.

[3] Zaid, Jamal, et al. "Zaid, Jamal, Mohammad Mahdi Farahani, and Tayeb A. Denidni. "Magneto-dielectric substrate-based microstrip antenna for RFID applications." IET Microwaves, Antennas \& Propagation 11.10 (2017): 1389-1392.

[4] Motevasselian, Alireza, and William G. Whittow. "Miniaturization of a Circular Patch Microstrip Antenna Using an Arc Projection." IEEE Antennas and Wireless Propagation Letters, vol. 16, 2017, pp. 517-520., doi:10.1109/lawp.2016.2586749.

[5] Zaid, Jamal, et al. "Miniaturized Multi-Port Microstrip Patch Antenna Using Metamaterial for Passive UHF RFID-Tag Sensor Applications." Sensors, vol. 19, no. 9, 2019, p. 1982., doi:10.3390/s19091982.

[6] Hamouda, Z., et al. "Magnetodielectric Nanocomposite Polymer-Based Dual-Band Flexible Antenna for Wearable Applications." IEEE Transactions on Antennas and Propagation, vol. 66, no. 7, 2018, pp. 32713277., doi:10.1109/tap.2018.2826573.

[7] Balanis, Constantine A., "Antenna Theory Analysis and Design," 2nd ed. Hoboken, NJ: Wiley, 2016.

[8] D. M. Pozar, "Microwave Engineering," 3rd ed. New York, NY, USA: Wiley, 2005.

[9] Mosallaei, H., and K. Sarabandi., "Magneto-Dielectrics in Electromagnetics: Concept and Applications." IEEE Transactions on Antennas and Propagation, vol. 52, no. 6, 2004, pp. 1558-1567., doi:10.11 09/tap.2004.829413.

[10] Niamien, Constant, et al. "Compact Expressions for Efficiency and Bandwidth of Patch Antennas Over Lossy Magneto-Dielectric Materials." IEEE Antennas and Wireless Propagation Letters, vol. 10, 2011, pp. 6366., doi:10.1109/lawp.2011.2107493.

[11] Hansen, R. C., and Mary Burke. "Antennas with Magneto-Dielectrics." Microwave and Optical Technology Letters, vol. 26, no. 2, 2000, pp. 7578., doi:10.1002/1098-2760(20000720)26:23.0.co;2-w.

[12] Rogers MAGTREX 555 datasheet. Rogers, www.rogerscorp.com/.

[13] Ikonen, P.m.t., et al. "Magnetodielectric Substrates in Antenna Miniaturization: Potential and Limitations." IEEE Transactions on Antennas and Propagation, vol. 54, no. 11, 2006, pp. 3391-3399., doi:10.1109/tap.2006.884303.

[14] Ho, Dinh Khanh, et al. "A Novel Dual-Band Rectenna for Ambient RF Energy Harvesting at GSM $900 \mathrm{MHz}$ and $1800 \mathrm{MHz}$." Advances in Science, Technology and Engineering Systems Journal, vol. 2, no. 3, 2017, pp. 612-616. 
[15] Abdulhadi, Abdulhadi Ebrahim, and Ramesh Abhari. "Multiport UHF RFID-Tag Antenna for Enhanced Energy Harvesting of Self-Powered Wireless Sensors." IEEE Transactions on Industrial Informatics 12.2 (2016): 801-808.

[16] Acher, O., and S. Dubourg. "Generalization of Snoek's Law to Ferromagnetic Films and Composites.” Physical Review B, vol. 77, no. 10, 2008, doi:10.1103/physrevb.77.104440.

[17] Rao, N., \& Dinesh Kumar, V. (2017). Miniaturization of Microstrip Patch Antenna for Satellite Communication: A Novel Fractal Geometry Approach. Wireless Personal Communications, 97(3), 3673-3683. https://doi.org/10.1007/s11277-017-4691-4.

[18] Kaur, A., Singh, G., \& Kaur, M. (2017). Miniaturized Multiband Slotted Microstrip Antenna for Wireless Applications. Wireless Personal Communications, 96(1), 441-453. https://doi.org/10.1007/s11277-0174177-4.

[19] M. Farahani, J. Zaid, T. A. Denidni and M. Nedil, "Miniaturized two dimensional circular polarized magneto-dielectric substrate antenna," 2016 IEEE International Symposium on Antennas and Propagation (APSURSI), Fajardo, PR, USA, 2016, pp. 1103-1104, doi: 10.1109/APS.2016.7696259.

[20]Zaid, Jamal, and Tayeb Ahmed Denidni. "Miniaturized CircularlyPolarized Patch Antenna Using an Artificial Metamaterial Substrate." Progress in Electromagnetics Research 109 (2021).

[21]Kumar, S. and Vishwakarma, D.K., "Miniaturisation of microstrip patch antenna using an artificial planar magneto-dielectric meta-substrate." IET Microwave Antennas Propagation," 2016, 10: 1235-1241. https://doi.org/10.1049/iet-map.2016.0044.

[22] Y. Liu, R. Raju, L. Shafai and C. Shafai, "A miniaturized artificial magneto-dielectric resonator antenna with split ring resonators," 201617 th International Symposium on Antenna Technology and Applied Electromagnetics (ANTEM), Montreal, QC, Canada, 2016, pp. 1-2, doi 10.1109/ANTEM.2016.7550234.

[23] J. Zaid, M. Mantash, A. Kesavan and T. A. Denidni, "Miniaturized Microstrip Patch Antenna Using Magneto-dielectric Substrate for RFID applications," 2018 IEEE International Symposium on Antennas and Propagation \& USNC/URSI National Radio Science Meeting, Boston, MA, USA, 2018, pp. 645-646, doi: 10.1109/APUSNCURSINRSM. 2018.8608675.

[24]Al Sharabati, Taher, and Bilal S. Taha. "Theoretical Modeling and Performance Assessment of the Effects of the Dissipation Factor (Loss Tangent) on Microstrip Patch Antennas Using Rogers' Kappa-438 Substrate." Antenna and Propagation (IRECAP) (2019): 447.

[25] Meddour, Fayçal, and Zohir Dibi. "An efficient small size electromagnetic energy harvesting sensor for low-DC-power applications." IET Microwaves, Antennas \& Propagation 11.4 (2016): 483-489.

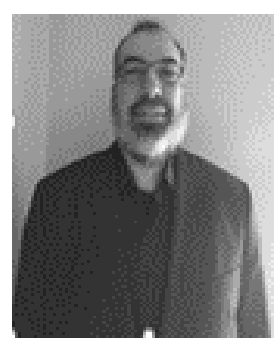

Taher AISharabati obtained his BSEE from St. Cloud State University, his MSEE from the University of Toledo, and his $\mathrm{PhD}$ in Electrical Engineering from the University of South Carolina. Between his MSEE and PhD, he worked in the industry for many years during which he worked at Motorola Inc. for about nine years. He was an Assistant and Associate Professor at Al Ahliyya Amman University from 02/2011 to 08/2019 in the Electronics and Communications Engineering Department. Currently, he is with the United States Patent and Trademark Office (USPTO). 